\title{
De quelles sources spécifiques a-t-on besoin en transformation de la matière?
}

\author{
J. C. Andre, M. L. Viriot et S. Corbel
}

GdR 0920 "Instrumentation Optique" CNRS et GRAPP-DCPR URA 328 CNRS ENSICINPL, BP. 451, 54001 Nancy Cedex, France

\section{Summary}

In order to develop activities related to light-matter interactions, diverse new sources are required, which can be coherent or not powerful or not, time resolved or not, etc... This paper will define, in an inductive way, some proposals for future.

\section{Résumé}

Le développement des activités en interactions lumière-matière requiert des sources lumineuses diverses, cohérentes ou non, puissantes ou non, résolues dans le temps ou non, etc... Le présent article définit de façon inductive quelques propositions d'actions pour l'avenir.

D'une manière générale, selon l'énergie des photons, on peut accéder à des excitations :

- rotationnelles et vibrationnelles de molécules,

- électroniques des électrons présents sur les orbitales les moins énergétiques,

- etc...

Dans ce contexte, en utilisant des flux lumineux plus ou moins intenses se sont développées des recherches dans les domaines reposant sur des processus à un ou plusieurs photons :

- photochimie,

- matériaux photochimiques,

- étude de la matière par des techniques spectroscopiques.

Dans toutes ces études on utilise généralement au moins l'une des quatre propriétés de la lumière que sont les résolutions :

- spectrales

- spatiales

- temporelles

- pour une moindre part, orientationnelles, associées à l'énergie ou à la puissance des sources lumineuses.

Transformer la matière inerte par une ou des interactions lumière(s)-matière correspond à un domaine tellement vaste qu'il est pratiquement exclu de pouvoir répondre sérieusement à la question posée dans le titre de cet article. En effet, selon que l'on effectue une absorption dans un milieu dense ou pas, on pourra avoir besoin d'une excitation spectrale fine ou non, d'une intensité lumineuse élevée ou non, etc..

Pour l'instant, le transformateur se contente de rencontres fortuites entre les sources lumineuses et les études à développer. Celles-ci ont d'ailleurs tendance à s'élargir grâce aux nouvelles sources UV (excimères, par exemple...) ou au doublage de fréquence. Alors a-t-on réellement besoin de sources UV et XUV nouvelles? 
Ceci est encore une fois le vieux problème de l' "œuf et de la poule". L'expérience montre que si l'on dispose de sources accordables, puissantes et d'un coût attractif, il est possible de trouver des applications desdites sources en transformation de la matière.

N'étant pas capables de proposer des solutions générales, nous avons préféré illustrer sur quelques exemples l'intérêt que peuvent (et doivent) posséder les sources UV et XUV pour promouvoir de nouvelles actions.

\section{SOURCES NOUVELLES ET PHOTOCHIMIE}

Tout d'abord, rappelons que l'essentiel des applications industrielles de la photochimie concerne des produits à basse ou moyenne valeur ajoutée (produits chlorés, sulfochlorés, sulfonés, ...) [1] [2]. Dans ces cas, les sources sont classiques : lampes à vapeur de mercure, dopées ou non.

En photochimie à un photon, le besoin de systèmes d'irradiation monochromatique se fait sentir dans les transformations de chimie fine [arômes, vitamines, médicaments] pour lesquelles le coût des réactifs n'est pas négligeable dans le coût global de la transformation.

Il s'agit en général de transformations successives du type :

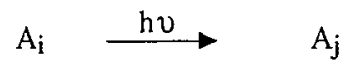

où des recouvrements des spectres d'absorption des molécules $A_{i}$ interviennent. Le choix d'un chemin optimal de réaction, visant la sélectivité maximale implique une évolution temporelle de(s) longueur(s) d'onde d'irradiation [3].

Ainsi, dans le cas de la transformation d'extraits d'arômes du houblon, cette sélectivité peut être $1 \mathrm{si}$ la longueur d'onde est supérieure à $360 \mathrm{~nm}$, inférieure jusqu'à moins de $20 \%$ en-dessous [1].

De même, pour la transformation photochimique de synthèse de la vitamine $\mathrm{D}$, l'emploi d'une irradiation laser, choisie pour sa "bonne" longueur d'onde conduit à un rendement de 0,8 au lieu des 0,3 obtenus à l'aide de lampes UV classiques à vapeur de mercure [4].

Ainsi, une bonne longueur d'onde d'excitation permet-elle d'améliorer les procédés photochimiques. Ce besoin de résolution spectrale se fait également sentir dans d'autres domaines connexes comme ceux liés aux séparations d'éléments traces comme les isotopes utilisés dans l'industrie nucléaire (Uranium, Tritium, Lithium, ...), les surfaces (isotopes du carbone pour des dépôts de type diamant), les terres rares, les métaux nobles, etc... [5].

Cette logique d'action vers des besoins industriels se retrouve dans un autre domaine, celui de la purification. La dégradation sélective d'impuretés dans des fluides est essentielle pour des applications nécessitant un haut degré de pureté comme la microélectronique, la catalyse chimique, etc... Pour ce faire, quelques études récentes montrent l'intérêt des excitations UV pour éliminer des polluants ou des poisons comme $\mathrm{H}_{2} \mathrm{~S}, \mathrm{PH}_{3}, \ldots$ [4].

En dehors de cet aspect résolution spectrale, on peut utiliser les sources UV et VUV pour participer à la mise au point des réactions nouvelles

- induites par un processus d'absorption multiphotonique suivi d'une trempe immédiate [6],

- induites photochimiquement conduisant à un rendement global (sélectivité) meilleur qu'à l'aide de techniques d'activation classiques.

C'est le cas en particulier de la réaction en chaîne longue de formation du chlorure de vinyle à partir du 1,2-dichloroéthane en phase gazeuse [4]. 


\section{II - SOURCES NOUVELLES ET MATERIAUX}

Les grands domaines d'applications des interactions lumière-matière dans la discipline matériaux concernent typiquement :

- la fabrication de poudres : céramiques, catalyseurs, ...

- les dépôts de surfaces : diamant, photopolymères, colles, métaux, ...

- l'ablation, le stockage d'information par photoérosion,

- la lithographie et la stéréophotolithographie, le stockage d'information,

- etc...

C'est encore une fois un domaine applicatif très large nécessitant soit de hauts degrés d'ionisation, soit de hautes températures, soit une bonne résolution spatiale... Dans ce cas également, la réponse à la question n'est pas simple.

Examinons toutefois une à une les différentes applications citées ci-dessus.

D'une manière générale la fabrication de poudres requiert de l'énergie permettant par exemple à partir d'un mélange $\mathrm{SiH}_{4} / \mathrm{CH}_{4}$ de fabriquer $\mathrm{SiC}$. En général, ce type de transformation est réalisé par irradiation laser IR à $10,6 \mu \mathrm{m}\left(\mathrm{CO}_{2}\right)$. Toutefois, la mise à disposition de sources UV de forte puissance qui autoriseraient des excitations dissociatives pourrait être une alternative à l'emploi des sources IR. Par ailleurs, dans l'UV lointain, il y aura pratiquement toujours absorption électronique permettant d'utiliser d'autres réactifs.

Les dépôts de surface correspondent également à une photochimie dissociative conduisant à des espèces atomiques comme le carbone, des métaux, des métallö̈des, etc... [4] [7] [8]. Le problème technique concerne peu la décomposition chimique induite par laser, mais plutôt la vitesse de nucléation associée au flux lumineux [7].

En revanche, pour la polymérisation induite par la lumière, il s'agit d'un processus associatif permettant localement le collage, le durcissement de vernis, etc... Le choix de la source n'est en général pas un problème fondamental à cause de l'effet amplificateur lié à l'existence de réactions en chaînes longues [1] [9].

On peut avec de la lumière favoriser un dépôt, on peut également favoriser une ablation. Dans ce cas, la profondeur et la largeur pénétrées dépendent de la longueur d'onde (résolution spatiale et coefficient d'absorption) ainsi que de la puissance ou de l'énergie de l'impulsion [10] [11] [12].

Pour le stockage d'informations par cette voie, on a intérêt à utiliser des longueurs d'onde courtes [1] [13] [14].

Cette même qualité de résolution se retrouve en lithographie et en stéréophotolithographie [15] où la transformation induite par la lumière doit être suffisamment propre, pour permettre la réalisation d'un bon contraste sur des distances de plus en plus courtes (inférieures à quelques dizièmes de $\mu \mathrm{m}$ ). Le développement de lasers VUV et XUV peut permettre des opérations nouvelles en lithographie et stéréophotolithographie laser grâce à des sources de faible coût (le synchroton du pauvre en quelque sorte...).

\section{SOURCES NOUVELLES ET ETUDE DE LA MATIERE}

Ce domaine peut être examiné sous différents angles, celui de l'instrumentation analytique et celui de la spectroscopie (chimie physique).

Si l'on considère tout d'abord le premier domaine, plusieurs tendances analytiques existent et se développent; il s'agit : 
- des capteurs à fibres optiques,

- des optodes actives,

- des spectroscopies IR, Raman, absorption, diffusion de lumière,

- de fluorescence et d'une manière générale d'émissions moléculaire et atomique,

- des lidars,

- des systèmes couplés laser-spectromètre,

- etc...

L'utilisation de sources UV lointain impose la mise en place de fibres transparentes dans ce domaine. De plus, dans ce domaine spectral, pratiquement tout absorbe, ce qui peut poser problèmes pour le développement des spectroscopies sauf pour des éléments traces (oxygène, ammoniac par exemple dans l'hydrogène, l'azote...).

En revanche, des sources UV lointain accordables peuvent permettre l'étude de l'ionisation de systèmes atomiques et moléculaires. La question posée concerne alors la sélectivité de cette technologie métrologique.

Toutefois, associée à d'autres spectroscopies comme la spectrométrie de masse, l'utilisation de sources pulsées de courtes longueurs d'onde peut permettre l'ionisation et par suite l'analyse chimique de substances volatiles directement, ou volatilisées par interaction lumière-surface.

Ainsi, pour des raisons techniques et spectroscopiques, l'analyse classique dans le domaine UV lointain présente en fait un intérêt modeste même si des niches existent [16] [17]. En revanche, les études de spectroscopie, avantageusement en phase gazeuse, sous faible pression, doivent permettre une meilleure compréhension de la matière grâce à des excitations résolues spectralement mais également résolues dans le temps [18].

Ce domaine est très vaste allant de l'étude des états excités électroniques aux spectroscopies (Hole burning, ...).

\section{CONCLUSIONS}

Nous avons rassemblé dans le tableau I les conclusions qualitatives correspondant aux domaines concernés en tranformation de la matière. Très clairement, toute source nouvelle peut avoir un intérêt.

Toutefois, si pour l'instant la photochimie exprime plutôt un besoin en terme de sources de photons incohérents, la demande peut être très différente pour les matériaux et les spectroscopies où il peut être avantageux d'utiliser les propriétés de stigmatisme des lasers.

De même, il peut être avantageux d'utiliser des longueurs d'onde précises et des résolutions temporelles (fluorescence résolue dans le temps par exemple).

Ainsi, la réponse à la question posée correspond à un "ramasse-tout" qui traduit cette conclusion simpliste mais réaliste :

"Donnez-nous de nouvelles sources UV et XUV cohérentes ou incohérentes, nous les utiliserons pour de bonnes études en transformation de la matière...". 
Tableau I

Essai de définition des besoins en sources UV et XUV pour la transformation de la matière.

\begin{tabular}{|c|c|c|c|}
\hline Domaine & Conditions & & Besoins \\
\hline Photochimie & $\begin{array}{l}\text { Phase condensée } \\
\text { Phase condensée } \\
\text { Phase gazeuse } \\
\text { Séparations }\end{array}$ & $\begin{array}{l}1 \text { photon } \\
\text { n photons } \\
1 \text { photon } \\
1 \text { photon } \\
\text { n photons }\end{array}$ & $\begin{array}{l}\text { Source accordable } \pm \text { qq } \mathrm{nm} \\
\text { Absorption, puissance } \\
\text { Source accordable, grande } \\
\text { résolution spectrale } \\
\text { Source accordable, grande } \\
\text { résolution spectrale }\end{array}$ \\
\hline Matériaux & $\begin{array}{l}\text { Poudres } \\
\text { Surfaces } \\
\text { Ablation } \\
\text { Lithographie } \\
\text { Stéréophotolithographie }\end{array}$ & $\begin{array}{l}1 / \mathrm{n} \text { photons } \\
1 / \mathrm{n} \text { photons } \\
\mathrm{n} \text { photons } \\
1 \text { photon }\end{array}$ & $\begin{array}{l}\text { Sources UV, puissance } \\
\text { Sources UV, puissance (?) } \\
\lambda \text { courte pour résolution } \\
\text { spatiale, puissance } \\
\lambda \text { courte, puissance (?) }\end{array}$ \\
\hline $\begin{array}{l}\text { Etude de la } \\
\text { matière }\end{array}$ & Spectroscopies & $\begin{array}{l}1 \text { photon } \\
\text { n photons }\end{array}$ & $\begin{array}{l}\text { Accordable, résolution } \\
\text { temporelle } \\
\text { Résolution temporelle, } \\
\text { puissance }\end{array}$ \\
\hline
\end{tabular}




\section{Bibliographie}

[1] VIRIOT, M L, ANDRE, J C et BRAUN, A, in "Industrial Photochemistry", CPIC-ENSIC Ed., NANCY (1990).

[2] ANDRE, J C, VIRIOT, M L et VILLERMAUX, J, Pure \& Appl. Chem., 58, (1986) 907-16.

[3] GEORGE, E, BOUCHY, A, ANDRE, J C et VIRIOT, M L, J. Photochem. \& Photobiol., A 48 (1989) 447-63.

[4] WOODIN, R, BOMSE, D S et RICE, G, Chem. \& Eng. News, $20-31$ (17 décembre 1990).

[5] QIU, L F, KANG, X H et WANG, T S, Separation Sci. \& Tech., 26 (1991) 199-221.

[6] LEMAIRE, F et STRINGAT, R, J. Phys., 48 (1987) 671.

[7] CELII, F G, NELSON, H H et PEHRSSON, P E, J. Mater. Res., 5 (1990) 2337-44.

[8] FERNANDEZ, A, MUNUERA, G, GONZALEZ-ELIPE, A R, ESPINOS, J P, HERRMANN, J M, PICHAT, P et LECLERCQ, C, Applied Catalysis, 57 (1990) 191-202

[9] HOYLE, C E, SPIE, 1213 (1990) 168-73.

[10] TAM, A C, LEUNG, W P et KRAJNOVICH, D, J. Appl. Phys., 69 (1991) 2072-75.

[11] MiSAWA, H, KITAMURA, N et MASUHARA, H, J. Am. Chem. Soc., 113 (1991) 7859-63

[12] DIENSTBIER, M, BENES, R, REJFIR, P et SLADKY, P, Appl. Phys., B51 (1990) $137-40$.

[13] BRANNON, J H, SCHOLL, D et KAY, E, Appl. Phys., A 52 (1991) 160-6.

[14] SRINIVASAN, R, Science, 234 (1986) 559.

[15] ANDRE, J C, in "Recherches en microtechniques : réalités et perspectives", Institut des Microtechniques et Cetehor Ed., Besançon (1992) 73-87.

[16] RZAZEWSKI, K, Acta Phys. Polon., A 78 (1990) 213-20.

[17] WAUTELET, M, GERMAIN, E et QUENON, P, Phys. Stat. Sol., 149 (1988) 465-75.

[18] PHILLIPS, L F, Search, 14 (1983) 23-8. 